\title{
Rho-Associated Protein Kinase (ROCK) Inhibitors Inhibit Survivin Expression and Sensitize Pancreatic Cancer Stem Cells to Gemcitabine
}

\author{
HIROYUKI TAKEDA ${ }^{1,2^{*}}$, MASASHI OKADA ${ }^{1 *}$, SHUHEI SUZUKI ${ }^{1,2}$, KENTA KURAMOTO $^{1}$, \\ HIROTSUGU SAKAKI ${ }^{1,3}$, HIKARU WATARAI ${ }^{1,4}$, TOMOMI SANOMACHI $^{1}$, \\ SHIZUKA SEINO ${ }^{1,5}$, TAKASHI YOSHIOKA ${ }^{2}$ and CHIFUMI KITANAKA ${ }^{1,5}$ \\ Departments of ${ }^{1}$ Molecular Cancer Science, ${ }^{2}$ Clinical Oncology, ${ }^{3}$ Obstetrics and Gynecology and \\ ${ }^{4}$ Second Department of Surgery, Yamagata University School of Medicine, Yamagata, Japan; \\ ${ }^{5}$ Research Institute for Promotion of Medical Sciences, Yamagata University Faculty of Medicine, Yamagata, Japan
}

\begin{abstract}
Background: Targeting pathways regulating survivin expression, which has been implicated in multidrug resistance of cancer cells, is a promising strategy to overcome cancer chemoresistance. To date, the role of rhoassociated protein kinases (ROCKs) in survivin expression remains largely unknown. Materials and Methods: The effects of ROCK inhibitors Y-27632 and fasudil on survivin expression and cell viability were determined by immunoblot analysis and dye exclusion, respectively, in PANC-1 CSLC, a cancer stem cell line derived from a serum-cultured, gemcitabine-sensitive pancreatic cancer cell line, PANC-1. Results: siRNA-mediated knockdown of survivin revealed that the gemcitabine resistance of PANC-1 CSLC was dependent on survivin expression. Both Y-27632 and fasudil, reduced survivin expression in PANC-1 CSLC cells and sensitized them to gemcitabine. ROCK inhibition also reduced survivin expression in various other human cancer cell lines. Conclusion: Small molecule inhibitor-mediated targeting of ROCK may be a viable strategy to overcome cancer chemoresistance through down-regulation of survivin.
\end{abstract}

Chemoresistance is a major obstacle that hampers successful management of cancer. Among cellular factors that

*These Authors contributed equally to this work.

Correspondence to: Chifumi Kitanaka and Masashi Okada, Department of Molecular Cancer Science, Yamagata University School of Medicine, Yamagata, 990-9585, Japan. Tel: +81 236285212, Fax: +81 236285215, e-mail: ckitanak@med.id.yamagata-u.ac.jp, mokada@med.id.yamagata-u.ac.jp

Key Words: ROK, chemosensitization, apoptosis, combination chemotherapy. contribute to the development of chemoresistance of cancer cells, survivin has been identified as one of the most crucial molecules. Survivin, an anti-apoptotic protein of the inhibitor of apoptosis protein (IAP) family, is not only expressed in a variety of human cancer types but also confers on cancer cells resistance to multiple chemotherapeutic agents including but not limited to gemcitabine, cisplatin, and paclitaxel. Thus, developing measures to inactivate survivin is expected as a pivotal step to overcome chemoresistance. Currently, a number of approaches for targeting survivin are under clinical development, however, small molecule inhibitors of survivin that are or are to be tested in clinical trials are still limited (1-3). In the present study, we aimed to identify novel small molecule drugs capable of targeting survivin expression.

\section{Materials and Methods}

Antibodies and reagents. Antibodies against sex determining region Y-box 2 (SOX2), survivin, glyceraldehyde 3-phosphate dehydrogenase (GAPDH), cleaved-caspase 3 (Asp175) and cleaved-poly(ADP-ribose) polymerase (PARP) (Asp214) were purchased from Cell Signaling Technology, Inc. (Beverly, MA, USA). Anti-E-cadherin (sc-8426) was from Santa Cruz Biotechnology, Inc. (Santa Cruz, CA, USA). Anti- $\beta$ actin (A1978) was from Sigma-Aldrich (St. Luis, MO, USA). ROCK inhibitors Y-27632 and fasudil hydrochloride were from Merck Millipore (Darmstadt, Germany) and Tokyo Chemical Industry Co., Ltd. (Tokyo, Japan), respectively. Y-27632 and fasudil were dissolved in water to prepare $10 \mathrm{mM}$ and $100 \mathrm{mM}$ stock solutions, respectively. Gemcitabine was purchased from Sigma-Aldrich and dissolved in dimethylsulfoxide to prepare a $1 \mathrm{mM}$ stock solution.

Cell culture. The human pancreatic cancer cell line PANC-1 was obtained from the Cell Resource Center for Biomedical Research, Institute of Development, Aging and Cancer, Tohoku University. PSN-1 was a kind gift from Dr. T. Yoshida at National Cancer Center Research Institute, who originally established this cell line from 
pancreatic adenocarcinoma tissue (4). A549 (a non-small cell lung cancer cell line) was obtained from the Riken BioResource Center (Tsukuba, Japan). A2780 (an ovarian cancer cell line) was a kind gift from Dr. T. Tsuruo (Institute of Molecular and Cellular Biosciences, University of Tokyo, Japan) and Drs. R.F. Ozols and T.C. Hamilton (the National Institutes of Health, USA) (5). SKOV-3 (an ovarian cancer cell line) and IMR90 normal human fetal lung fibroblasts were purchased from the American Type Culture Collection (ATCC, Manassas, VA, USA). RMG-1 (an ovarian cancer cell line) was kindly provided by Dr S. Nozawa and Dr D. Aoki at Keio University, Japan (6). SKOV-3 was maintained in M199:105 medium, a 1:1 mixture of M199 and MCDB105 media supplemented with 10\% fetal bovine serum (FBS; Sigma) (7-9). A549, RMG-1, A2780 and PSN-1 were maintained in Dulbecco's modified Eagle's medium (DMEM)/F12 medium supplemented with 10\% FBS (4, 8). IMR90 was maintained in DMEM supplemented with $10 \%$ FBS. These culture media were further supplemented with $100 \mathrm{U} / \mathrm{ml}$ penicillin and $100 \mu \mathrm{g} / \mathrm{ml}$ streptomycin.

The establishment of cancer stem cells used in this study (PANC1 CSLC, A549 CSLC, and GS-Y03) has been described elsewhere (10-12). These cells were maintained under the monolayer stem cell culture condition as previously reported (10-12). Briefly, cells were cultured on collagen-I-coated dishes (IWAKI, Tokyo, Japan) in the stem cell culture medium [DMEM/F12 medium supplemented with 1\% B27 (Thermo Fisher Scientific, Waltham, MA USA), 20 ng/ml epidermal growth factor (EGF) and fibroblast growth factor (FGF2) (Peprotech Inc., Rocky Hill, NJ, USA), D-(+)-glucose (final concentration, $26.2 \mathrm{mM}$ ), L-glutamine (final concentration, $4.5 \mathrm{mM}$ ), $100 \mathrm{units} / \mathrm{ml}$ penicillin, and $100 \mu \mathrm{g} / \mathrm{ml}$ streptomycin]. Stem cell culture medium was changed approximately every 3 days, and EGF and FGF2 were added to the culture medium every day. The authenticity of the cells used in this study was verified by the genotyping of short tandem repeat (STR) loci (Bio-Synthesis Inc., Lewisville, TX, USA) followed by comparison to the ATCC STR database (http://www.atcc.org/STR_Database.aspx) for Human Cell Lines. All IMR90 experiments were performed using low passage number $(<8)$ cells.

Gene silencing by siRNA. siRNAs against human survivin (BIRC5; \#1 HSS HSS179403, \#2 HSS179404, \#3 HSS179405) and Medium GC Duplex \#2 of Stealth RNAi ${ }^{\mathrm{TM}}$ siRNA Negative Control Duplexes (as a non-targeting control for siRNA experiments) were purchased from Thermo Fisher Scientific. PANC-1 CSLC cells were transiently transfected with siRNAs against survivin (siSurvivin) or with a control siRNA (siControl) and were treated on the next day with or without $0.5 \mu \mathrm{M}$ gemcitabine for 3 days. The cells were then subjected to immunoblot analysis of survivin, caspase-3 and poly(ADP-ribose) polymerase (PARP) at 4 days after transfection and to cell viability assay using propidium iodide (PI). Transfection of siRNAs was performed using Lipofectamine RNAiMAX (Thermo Fisher Scientific) according to the manufacturer's instructions.

Cell viability assay. Viable and dead cells were identified by their ability and inability to exclude vital dyes, respectively (13-15). Briefly, cells were stained with $0.2 \%$ trypan blue for $1 \mathrm{~min}$ at room temperature, and the numbers of viable and dead cells were determined using a hemocytometer. The percentage of dead cells was defined as $100 \times$ [number of dead cells/(the number of viable + dead cells)]. Alternatively, cells were incubated in situ with propidium iodide (PI; $1 \mu \mathrm{g} / \mathrm{ml})$ and Hoechst $33342(10 \mu \mathrm{g} / \mathrm{ml})$ for
10 min at $37^{\circ} \mathrm{C}$ in a $\mathrm{CO}_{2}$ incubator to stain the dead cells and nuclei, respectively. Subsequently, the numbers of PI- and Hoechstpositive cells were scored under a fluorescence microscope (CKX41; OLYMPUS, Tokyo, Japan), and the percentage of PIpositive cells (dead cells) relative to Hoechst-positive cells (total cells) was determined.

Immunoblot analysis. Immunoblot analysis was conducted as previously described $(11,13,15,16)$. Cells were washed with icecold phosphate-buffered saline and lysed in RIPA buffer [10 mM Tris- $\mathrm{HCl}$ ( $\mathrm{pH} 7.4$ ), $0.1 \%$ sodium dodecyl sulfate (SDS), $0.1 \%$ sodium deoxycholate, $1 \% \mathrm{NP}-40,150 \mathrm{mM} \mathrm{NaCl}, 1 \mathrm{mM}$ EDTA, 1.5 $\mathrm{mM} \mathrm{Na} \mathrm{VO}_{4}, 10 \mathrm{mM} \mathrm{NaF}, 10 \mathrm{mM}$ sodium pyrophosphate, $10 \mathrm{mM}$ sodium $\beta$-glycerophosphate and $1 \%$ protease inhibitor cocktail set III (Merck Millipore)]. After centrifugation for $10 \mathrm{~min}$ at $14,000 \times$ $g$ at $4^{\circ} \mathrm{C}$, the supernatants were recovered as cell lysates, and the protein concentration of the cell lysates was determined by a BCA protein assay kit (Pierce Biotechnology Inc., Rockford, IL, USA). Cell lysates containing equal amounts of protein were separated by SDS-polyacrylamide gel electrophoresis and transferred to a polyvinylidene difluoride membrane. The membrane was probed with a primary antibody and then with an appropriate horseradish peroxidase-conjugated secondary antibody, according to the protocol recommended by the manufacturer of each antibody. Immunoreactive bands were visualized using Immobilon Western Chemiluminescent HRP Substrate (Millipore).

Statistical analysis. Results are expressed as means and standard deviation (SD), and differences were compared using the two-tailed Student's $t$-test. $p$-Values of less than 0.05 were considered statistically significant.

\section{Results}

Survivin overexpression confers gemcitabine resistance on pancreatic cancer stem cells. We previously demonstrated that PANC-1 CSLC, a cancer stem cell line established from a serum-cultured pancreatic cancer cell line PANC-1 (characterized by the expression of epithelial differentiation marker E-cadherin but not SOX2, a stem cell marker) and characterized by the expression of SOX2 but not E-cadherin, is highly resistant to gemcitabine-induced cell death compared to the original PANC-1 from which it was derived (15). While searching for cell death-related molecules that are differentially expressed or activated in these cell lines in an attempt to determine the molecular alterations responsible for the cell death resistance of PANC-1 CSLC, we found that PANC-1 CSLC expressed a much higher level of survivin than PANC-1 did (Figure 1). To determine whether survivin overexpression is involved in the gemcitabine resistance of PANC-1 CSLC, we tested the effect of survivin knockdown on the resistance/sensitivity of PANC-1 CSLC cells to gemcitabine. Although survivin knockdown by itself increased cell death to some extent, gemcitabine-induced cell death was significantly and considerably promoted after survivin knockdown, suggesting that survivin has a role in gemcitabine resistance of PANC-1 CSLC cells (Figure 2). 


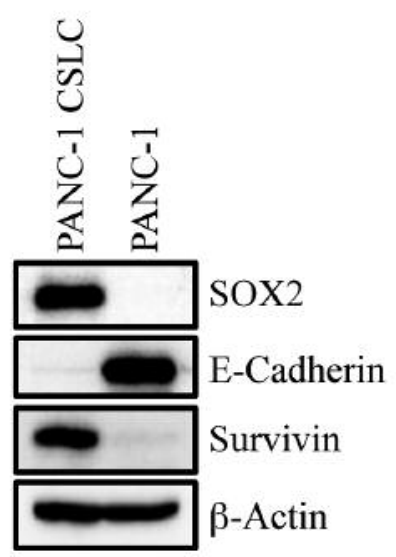

Figure 1. Survivin overexpression by a gemcitabine-resistant cancer stem cell line (PANC-1 CSLC) derived from the gemcitabine-sensitive pancreatic cancer cell line PANC-1. PANC-1 and PANC-1 CSLC cells were examined for the expression of survivin as well as for sex determining region Y-box2 (SOX2) (a stem cell marker) and E-cadherin (an epithelial differentiation marker) by immunoblot analysis.

ROCK inhibitors reduce survivin expression and sensitize pancreatic cancer stem cells to gemcitabine. With the intention of identifying drugs which therapeutically target survivin expression in order to overcome chemoresistance associated with survivin overexpression, we screened a series of small-molecule modulators of signaling pathways against survivin expression in PANC-1 CSLC cells (data not shown). As a result, we discovered that Y-27632, a ROCK inhibitor, efficiently inhibits survivin expression in PANC-1 CSLC cells. Y-27632 reduced the expression of survivin in PANC-1 CSLC cells in a concentration-dependent manner (Figure 3A). At 10 $\mu \mathrm{M}$, a concentration at which $\mathrm{Y}-27632$ did not impair the viability of normal cells (see Figure 4A), the reduction of survivin expression became evident 3 days after Y-27632 treatment (Figure 3C). Prompted by the identification of Y-27632 as a modulator of survivin expression, we tested another ROCK inhibitor fasudil, which is clinically approved for the treatment of cerebral vasospasm following subarachnoid hemorrhage (17). Similarly to Y-27632, fasudil reduced survivin expression in PANC-1 CSLC cells in a concentration-dependent manner and at a concentration $(25 \mu \mathrm{M})$ not toxic to normal cells (Figure 3B and D see also Figure 4B).

We then asked whether these ROCK inhibitors capable of inhibiting survivin expression promote gemcitabine-induced cell death in PANC-1 CSLC cells. To determine the nontoxic concentrations of Y-27632, we treated IMR90 normal human fibroblasts with increasing concentrations of Y-27632 and confirmed that IMR90 cells tolerated up to $10 \mu \mathrm{M}$ (Figure 4A). Having confirmed that Y-27632 alone also does not increase cell death in PANC-1 CSLC cells at this concentration (10 $\mu \mathrm{M})$ (Figure 4D), we examined the sensitivity of PANC-1 CSLC cells to gemcitabine in the presence and absence of Y-27632 treatment. At $0.5 \mu \mathrm{M}$, gemcitabine alone caused only modest $(\sim 10 \%)$ death of PANC-1 CSLC cells. However, when PANC-1 CSLC cells were first treated with Y-27632 followed by treatment with $0.5 \mu \mathrm{M}$ gemcitabine, cell death was remarkably increased compared to gemcitabine treatment alone (Figure 4D), demonstrating that Y-27632 sensitizes PANC-1 CSLC cells to gemcitabine. Importantly, the identical combination treatment did not increase death of IMR90 cells, suggesting that the combination is specifically cytotoxic to cancer cells while sparing normal cells (Figure 4C). Of note, the expression of cleavage products of caspase 3 and PARP also increased in parallel with cell death, which suggests that apoptosis may be involved as a cell death mechanism (Figure 4F). Essentially similar results were obtained when fasudil was used in the place of Y-27632 (Figure 4B, C, E and G). Together, these results suggest that ROCK inhibitors sensitize PANC-1 CSLC cells to gemcitabine most likely through down-regulation of survivin, without increasing the toxicity of gemcitabine to normal cells.

ROCK inhibition causes down-regulation of endogenously overexpressed survivin in various human cancer cell lines. Our results thus far suggested that survivin expression in PANC-1 CSLC cells is dependent on ROCK. To determine whether survivin expression is sensitive to ROCK inhibition uniquely in PANC-1 CSLC cells or widely in human cancer cells including non-cancer stem cells, we treated human cancer cell lines derived from different cancer types that overexpress survivin with Y-27632. Strikingly, ROCK inhibition caused significant decrease in survivin expression in all cell lines examined (Figure 5), suggesting that ROCK may be involved in survivin overexpression in a variety of human cancer cells.

\section{Discussion}

Survivin, which confers multidrug resistance on cancer cells, is overexpressed in cancer cells and not in terminally differentiated adult tissues. Survivin is therefore an ideal target in oncology therapeutics, and control of the expression or function of survivin has been considered a prerequisite for successful cancer chemotherapy. Currently, methods to therapeutically target survivin are being explored and developed actively, yet are still limited (1-3). In this study, we demonstrated, for the first time to the best of our knowledge, that ROCK inhibition by use of smallmolecule inhibitors successfully reduces expression of endogenous survivin overexpressed in pancreatic cancer stem cell line PANC-1 CSLC as well as in a variety of human cancer cell lines. 

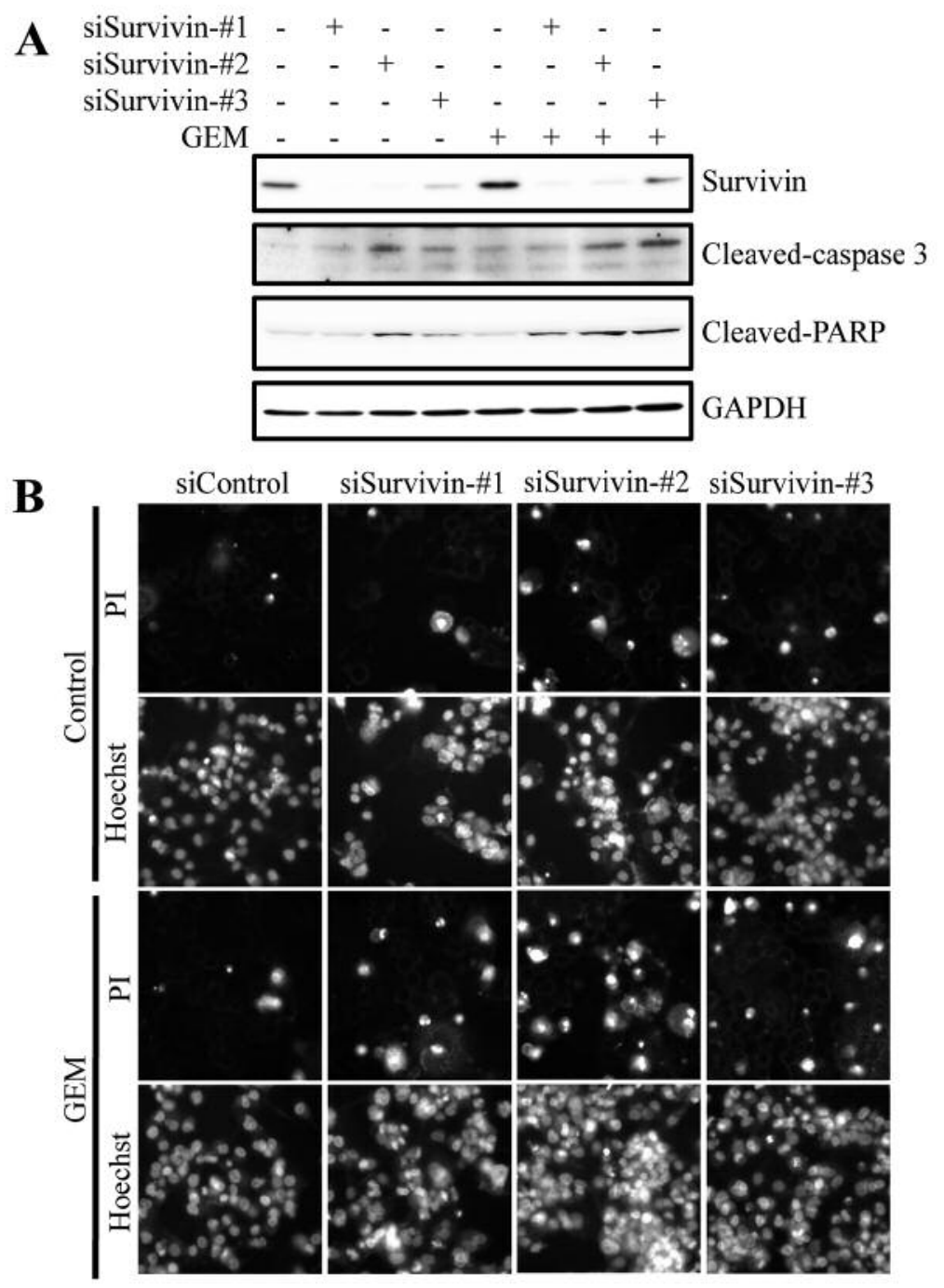

C
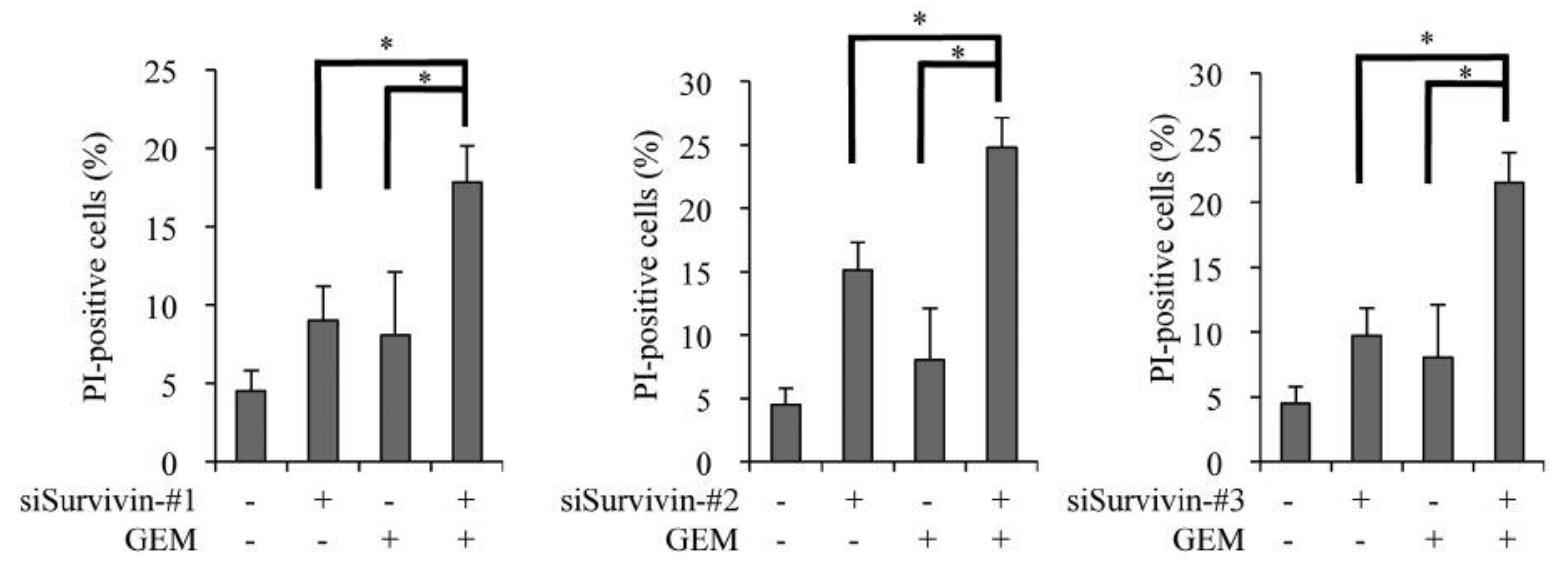

Figure 2. Gemcitabine resistance of PANC-1 CSLC cells is dependent on survivin expression. PANC-1 CSLC cells transiently transfected with siRNAs against survivin (siSurvivin) or with a control siRNA (siControl) were treated on the next day with or without gemcitabine (GEM, $0.5 \mu M)$ for 3 days. The cells were then subjected to immunoblot analysis of survivin, caspase-3 and poly(ADP-ribose) polymerase (PARP) at 4 days after transfection (A) or to cell viability assay using propidium iodide $(P I)(B$ and $C)$. B: Representative fluorescence images of PI- and Hoechst-positive cells. C: The percentage of PI-positive cells (dead cells) relative to Hoechst-positive cells (total cells). Values in the graphs represent means $\pm S D$ from triplicate samples of a representative experiment repeated with similar results. ${ }^{*} p<0.05$. GAPDH: Glyceraldehyde 3-phosphate dehydrogenase. 

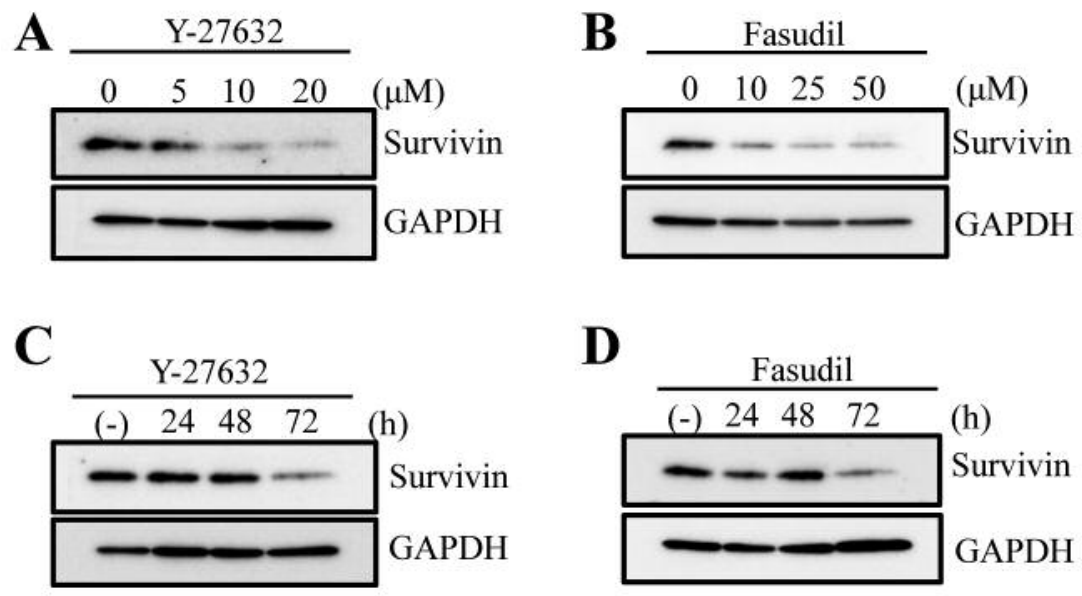

Figure 3. Suppression of survivin expression in PANC-1 CSLC cells by rho-associated protein kinase (ROCK) inhibitors. PANC-1 CSLC cells were treated with Y-27632 (A) or fasudil (B) at different concentrations for 3 days and at $10 \mu M$ Y-27632 (C) or $25 \mu M$ fasudil (D) for different time periods $(24-72 \mathrm{~h})$ or without these drugs for $72 \mathrm{~h}(-)$. The cells were then subjected to immunoblot analysis of survivin expression. GAPDH: Glyceraldehyde 3-phosphate dehydrogenase.

A previous study demonstrated that $\mathrm{RhoC}$ is overexpressed in ovarian cancer and that RhoC knockdown reduced survivin expression in ovarian cancer cell line OVCAR-3 (18). Although the downstream signaling pathway linking RhoC and survivin remained to be shown, our results suggest that ROCK may be involved therein given that ROCK can be activated by RhoC (19). Intriguingly enough, we have shown in this study that survivin expression is reduced upon ROCK inhibition in three ovarian cancer cell lines (SKOV-3, RMG-1, and A2780). It would be interesting to speculate that overexpression of RhoC might be involved in survivin overexpression through ROCK activation in these ovarian cancer cell lines. Another study demonstrated that endothelin-1 (ET1) treatment renders normal human fibroblasts resistant to apoptosis by inducing the expression of survivin in a ROCK-dependent manner (20). To date, the role and mechanism of ET1 signaling in survivin overexpression in cancer cells remain largely unknown. However, since the ET1 pathway is aberrantly activated in various human cancer types (21), our results may give rise to a novel, intriguing possibility that ET1 signalingdependent activation of ROCK plays a significant role in the overexpression of survivin by cancer cells.

We demonstrated in this study that survivin knockdown sensitizes PANC-1 CSLC cells to gemcitabine on the one hand and that ROCK inhibitors reduce survivin expression in PANC-1 CSLC cells sensitizing them to gemcitabine on the other. While these results strongly suggest that reduced survivin expression contributes to gemcitabine sensitization by ROCK inhibitors, the results do not necessarily exclude the possibility that ROCK inhibitors may sensitize cells to gemcitabine through other mechanisms. Although details of the mechanism remain to be shown, it is clear from our results that ROCK inhibitors are of use in overcoming chemoresistance of cancer cells. Since survivin has been associated with resistance to cisplatin, paclitaxel, mitomycin $\mathrm{C}$, and docetaxel in addition to gemcitabine (1), the combination of ROCK inhibitors with these chemotherapeutic agents may be a rational approach to treat cancer resistant to these agents. In support of this idea, the benefit of combination with Y-27632 was documented for cisplatin in a previous study (22). Our promising data together with those of others thus appear to warrant preclinical animal studies using Y-27632 or fasudil as a chemosensitizer, but it would be important to bear in mind that a large number of novel ROCK inhibitors are being developed with some already in clinical trials for other purposes (17). Further in vitro studies examining other ROCK inhibitors for their efficacy in inhibiting survivin expression and in chemosensitization will help determine which ROCK inhibitor should be chosen and advanced to preclinical animal studies to evaluate its in vivo effect as a chemosensitizer. In this regard, ROCK inhibitors with a known safety profile in humans, such as fasudil, may be preferred.

In conclusion, the present study demonstrated for the first time that ROCK inhibitors are promising chemosensitizers that can target survivin expression in a variety of human cancer cells. ROCK inhibitors in combination with chemotherapeutic agents may become a rational and viable strategy to treat cancer with multidrug resistance associated with survivin overexpression. 

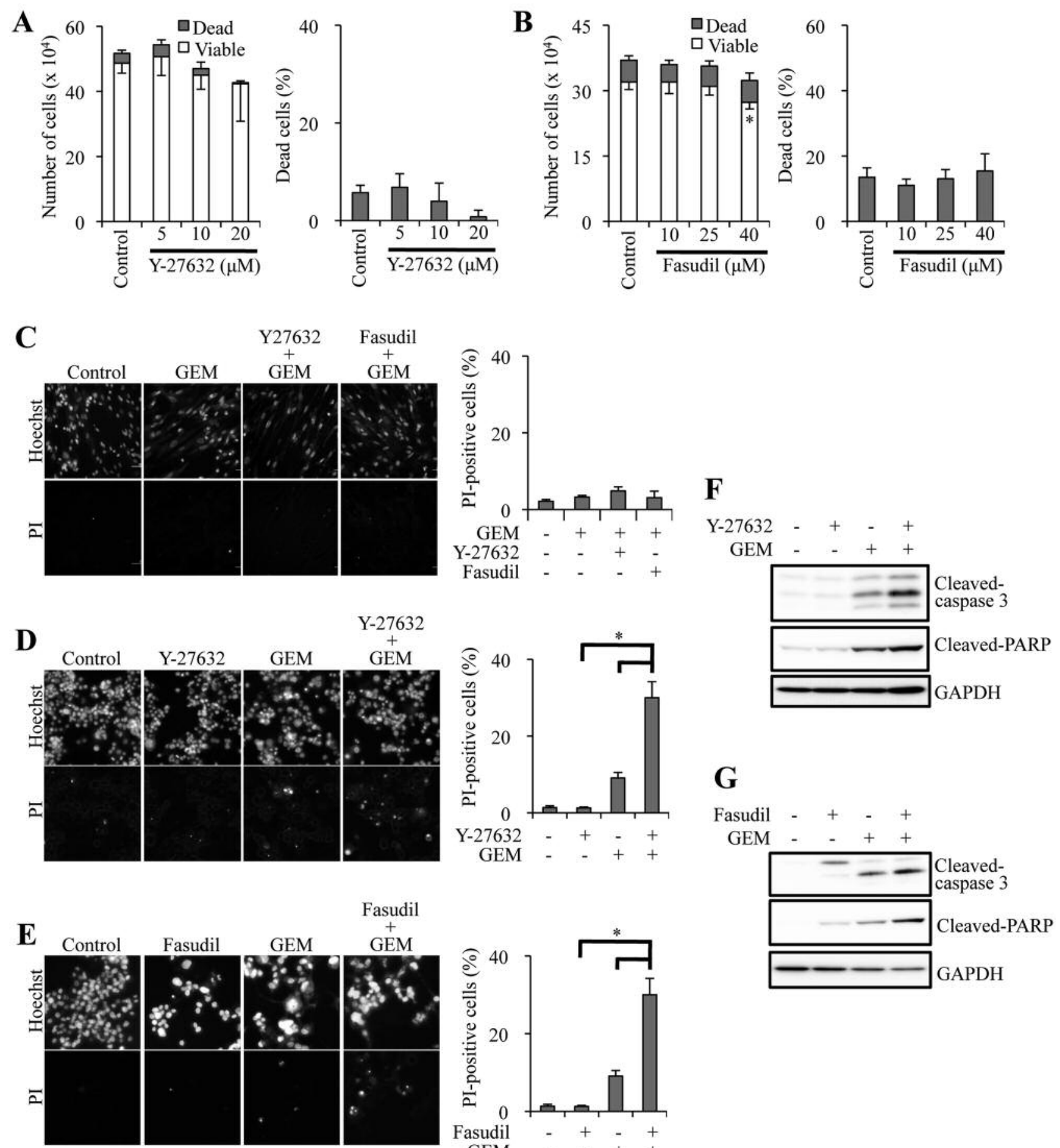

Fasudil
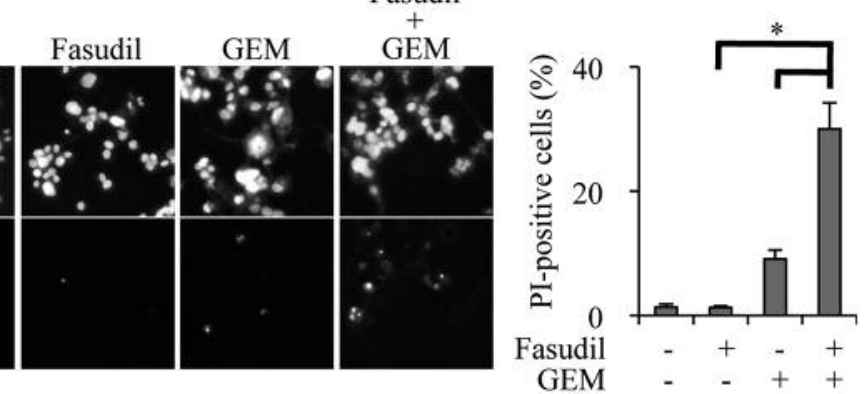

Figure 4. Rho-associated protein kinase (ROCK) inhibitors promote gemcitabine-induced apoptosis selectively in cancer stem cells. IMR90 normal human fibroblasts were treated without (Control) or with different concentrations of Y-27632 (A) or fasudil (B) for 3 days then subjected to cell viability assay using trypan blue. The numbers of viable and dead cells and the percentage of dead cells are shown in the left and right panels, respectively. IMR90 (C) and PANC-1 CSLC (D-G) cells were pretreated with or without Y-27632 (10 $\mu M, C, D, F)$ or fasudil (25 $\mu M, C, E, G)$ for 3 days and subsequently treated with or without gemcitabine $(G E M, 0.5 \mu M)$ for 3 days in the presence of the pretreatment drugs, as indicated. The cells were then subjected to cell viability assay using propidium iodide (PI) (C-E) or to immunoblot analysis of cleaved-caspase 3 and poly(ADPribose) polymerase (PARP) $(F, G)$. C-E: Left panels show representative fluorescence images of PI- and Hoechst-positive cells. A-E: Values in the graphs represent means $\pm S D$ from triplicate samples of a representative experiment repeated with similar results. *p $<0.05$. GAPDH: Glyceraldehyde 3-phosphate dehydrogenase. 


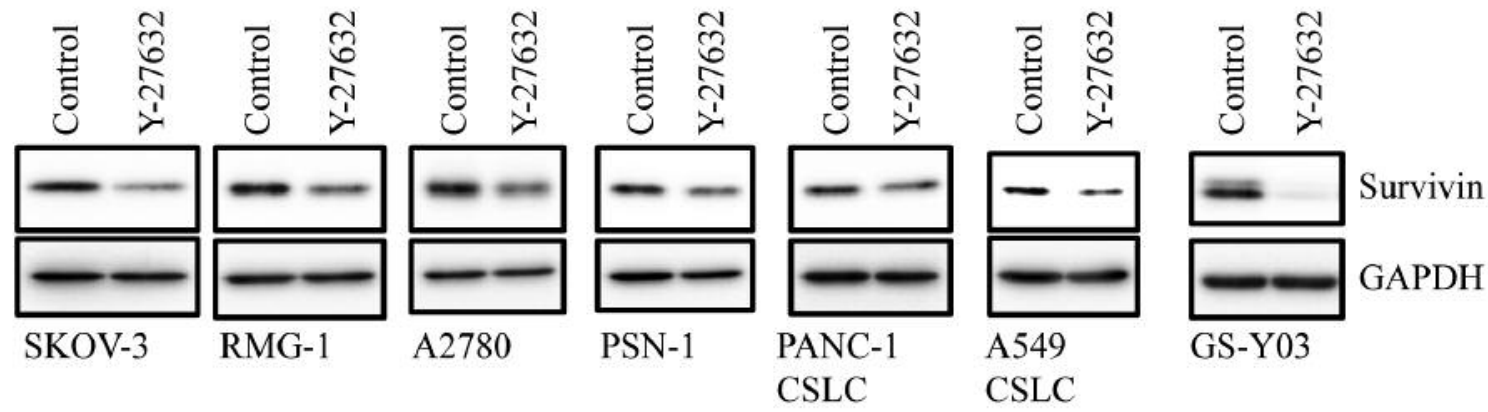

Figure 5. Rho-associated protein kinase (ROCK) inhibition results in the reduction of survivin expression in various human cancer cells. Cells were treated without (Control) or with Y-27632 (10 $\mu \mathrm{M})$ for 3 days then examined for survivin expression by immunoblot analysis. GAPDH: Glyceraldehyde 3-phosphate dehydrogenase.

\section{Conflicts of Interest}

The Authors declare no conflict of interest.

\section{Acknowledgements}

This work was supported by Grants-in-Aid for Scientific Research, for Challenging Exploratory Research, and for Young Scientists from the Ministry of Education, Culture, Sports, Science and Technology of Japan.

\section{References}

1 Fatemian T, Othman I and Chowdhury EH: Strategies and validation for siRNA-based therapeutics for the reversal of multidrug resistance in cancer. Drug Discov Today 19: 71-78, 2014.

2 Garg H, Suri P, Gupta JC, Talwar GP and Dubey S: Survivin: a unique target for tumor therapy. Cancer Cell Int 16: 49, 2016.

3 Singh N, Krishnakumar S, Kanwar RK, Cheung CH and Kanwar JR: Clinical aspects for survivin: a crucial molecule for targeting drug-resistant cancers. Drug Discov Today 20: 578-587, 2015.

4 Yamada H, Yoshida T, Sakamoto H, Terada M and Sugimura T: Establishment of a human pancreatic adenocarcinoma cell line (PSN-1) with amplifications of both $c-M Y C$ and activated $c-K I-$ $R A S$ by a point mutation. Biochem Biophys Res Commun 140: 167-173, 1986.

5 Hamilton TC, Winker MA, Louie KG, Batist G, Behrens BC, Tsuruo T, Grotzinger KR, McKoy WM, Young RC and Ozols RF: Augmentation of adriamycin, melphalan, and cisplatin cytotoxicity in drug-resistant and -sensitive human ovarian carcinoma cell lines by buthionine sulfoximine mediated glutathione depletion. Biochem Pharmacol 34: 2583-2586, 1985.

6 Suzuki N, Aoki D, Tamada Y, Susumu N, Orikawa K, Tsukazaki K, Sakayori M, Suzuki A, Fukuchi T, Mukai M, Kojima-Aikawa K, Ishida I and Nozawa S: HMOCC-1, a human monoclonal antibody that inhibits adhesion of ovarian cancer cells to human mesothelial cells. Gynecol Oncol 95: 290-298, 2004.

7 Liu Z, Yamanouchi K, Ohtao T, Matsumura S, Seino M, Shridhar V, Takahashi T, Takahashi K and Kurachi H: High levels of Wilms' tumor 1 (WT1) expression were associated with aggressive clinical features in ovarian cancer. Anticancer Res 34: 2331-2340, 2014.

8 Seino M, Okada M, Sakaki H, Takeda H, Watarai H, Suzuki S, Seino S, Kuramoto K, Ohta T, Nagase S, Kurachi H and Kitanaka C: Time-staggered inhibition of JNK effectively sensitizes chemoresistant ovarian cancer cells to cisplatin and paclitaxel. Oncol Rep 35: 593-601, 2016.

9 Yamanouchi K, Ohta T, Liu Z, Oji Y, Sugiyama H, Shridhar V, Matsumura S, Takahashi T, Takahashi K and Kurachi H: The Wilms' tumor gene WT1 - 17AA/- KTS splice variant increases tumorigenic activity through up-regulation of vascular endothelial growth factor in an in vivo ovarian cancer model. Transl Oncol 7: 580-589, 2014.

10 Matsuda K, Sato A, Okada M, Shibuya K, Seino S, Suzuki K, Watanabe E, Narita Y, Shibui S, Kayama T and Kitanaka C: Targeting JNK for therapeutic depletion of stem-like glioblastoma cells. Sci Rep 2: 516, 2012.

11 Okada M, Shibuya K, Sato A, Seino S, Suzuki S, Seino M and Kitanaka C: Targeting the K-Ras-JNK axis eliminates cancer stem-like cells and prevents pancreatic tumor formation. Oncotarget 5: 5100-5112, 2014.

12 Okada M, Shibuya K, Sato A, Seino S, Watanabe E, Suzuki S, Seino $M$ and Kitanaka C: Specific role of JNK in the maintenance of the tumor-initiating capacity of A549 human nonsmall cell lung cancer cells. Oncol Rep 30: 1957-1964, 2013.

13 Okada M, Kuramoto K, Takeda H, Watarai H, Sakaki H, Seino S, Seino M, Suzuki S and Kitanaka C: The novel JNK inhibitor AS602801 inhibits cancer stem cells in vitro and in vivo. Oncotarget 7: 27021-27032, 2016.

14 Okada M, Sato A, Shibuya K, Watanabe E, Seino S, Suzuki S, Seino M, Narita Y, Shibui S, Kayama T and Kitanaka C: JNK contributes to temozolomide resistance of stem-like glioblastoma cells via regulation of MGMT expression. Int J Oncol 44: 591$599,2014$.

15 Suzuki S, Okada M, Shibuya K, Seino M, Sato A, Takeda H, Seino S, Yoshioka $\mathrm{T}$ and Kitanaka C: JNK suppression of chemotherapeutic agents-induced ROS confers chemoresistance on pancreatic cancer stem cells. Oncotarget 6: 458-470, 2015.

16 Shibuya K, Okada M, Suzuki S, Seino M, Seino S, Takeda H and Kitanaka C: Targeting the facilitative glucose transporter GLUT1 inhibits the self-renewal and tumor-initiating capacity of cancer stem cells. Oncotarget 6: 651-661, 2015. 
17 Wei L, Surma M, Shi S, Lambert-Cheatham N and Shi J: Novel Insights into the roles of rho kinase in cancer. Arch Immunol Ther Exp 64: 259-278, 2016.

18 Zhao Y, Zheng HC, Chen S, Gou WF, Xiao LJ and Niu ZF: The role of RhoC in ovarian epithelial carcinoma: a marker for carcinogenesis, progression, prognosis, and target therapy. Gynecol Oncol 130: 570-578, 2013.

19 Julian L and Olson MF: Rho-associated coiled-coil containing kinases (ROCK): structure, regulation, and functions. Small GTPases 5: e29846, 2014.

20 Horowitz JC, Ajayi IO, Kulasekaran P, Rogers DS, White JB, Townsend SK, White ES, Nho RS, Higgins PD, Huang SK and Sisson TH: Survivin expression induced by endothelin-1 promotes myofibroblast resistance to apoptosis. Int J Biochem Cell Biol 44: 158-169, 2012.
21 Rosano L, Spinella F and Bagnato A: Endothelin 1 in cancer: biological implications and therapeutic opportunities. Nat Rev Cancer 13: 637-651, 2013.

22 Ohta T, Takahashi T, Shibuya T, Amita M, Henmi N, Takahashi $\mathrm{K}$ and Kurachi $\mathrm{H}$ : Inhibition of the Rho/ROCK pathway enhances the efficacy of cisplatin through the blockage of hypoxia-inducible factor-1alpha in human ovarian cancer cells. Cancer Biol Ther 13: 25-33, 2012.

Received September 19, 2016

Revised October 5, 2016

Accepted October 10, 2016 